\title{
Pour une génétique de la littérature d'enfance et de jeunesse
}

Christine Collière-Whiteside et Karine Meshoub-Manière

\section{(2) OpenEdition}

1 Journals

Édition électronique

URL : http://journals.openedition.org/genesis/3818

DOI : 10.4000/genesis.3818

ISSN : 2268-1590

Éditeur :

Presses universitaires de Paris Sorbonne (PUPS), Société internationale de génétique artistique littéraire et scientifique (SIGALES)

Édition imprimée

Date de publication : 1 juin 2019

Pagination : 7-19

ISSN : 1167-5101

Référence électronique

Christine Collière-Whiteside et Karine Meshoub-Manière, « Pour une génétique de la littérature d'enfance et de jeunesse », Genesis [En ligne], 48 | 2019, mis en ligne le 01 juin 2019, consulté le 25 janvier 2021. URL : http://journals.openedition.org/genesis/3818 ; DOI : https://doi.org/10.4000/ genesis.3818 


\title{
Pour une génétique de la littérature d'enfance et de jeunesse
}

\author{
Christine Collière-Whiteside et Karine Meshoub-Manière
}

C

omme la bande dessinée $(B D)$, la littérature d'enfance et de jeunesse a connu un processus de légitimation (Boltanski ${ }^{1}$ ) relativement récent qui est à la croisée de différentes disciplines (histoire, histoire de l'art, littérature comparée, sciences de l'éducation, psychologie, culture médiatique, étude du jeu...). Depuis les travaux pionniers de Marc Soriano, de Jean Perrot et d'Isabelle Nières-Chevrel ${ }^{2}$ dans les années soixante-dix, elle s'est constituée en objet d'étude et de recherche, qui s'enseigne dans des masters spécialisés.

Pourtant, cette littérature demeure encore largement absente du champ de la génétique textuelle, bien que celle-ci se soit intéressée à des genres littéraires dits mineurs, avec le numéro de Recto/Verso consacré aux «Mauvais genres ${ }^{3}$ » et le numéro de Genesis sur la BD ${ }^{4}$, dont les corpus appartiennent pour partie à la littérature pour la jeunesse. Si cette dernière ouvre à la génétique textuelle des corpus supplémentaires, riches de genres variés, de supports multiples et de dispositifs auctoriaux divers, lui offre-t-elle pour autant un nouveau champ de recherche? Sauf à s'intéresser exclusivement à l'album qui, comme la BD, est un iconotexte (pour reprendre le terme de Michael Nerlich) ${ }^{5}$, et pose des questions spécifiques sur le rapport entre textes et images au cours du processus créatif, la littérature pour la jeunesse n'est pas un genre. Parfois très codifiée, beaucoup plus audacieuse ailleurs, elle ne se caractérise ni par une forme ni par un média spécifique qui poseraient des questions génétiques propres. Tous les genres littéraires y sont représentés : poésie, théâtre, récit (englobant aussi bien les contes, les romans, courts ou longs, les récits d'aventure, les récits policiers, etc.), dans des registres variés (textes comiques ou tragiques, textes réalistes ou fantastiques), que ce soit

1. Luc Boltanski, «La constitution du champ de la bande dessinée», Actes de la recherche en sciences sociales, vol. I, $\mathrm{n}^{\circ}$ 1, janvier 1975. Voir aussi Max Butlen, « La littérature de jeunesse, chronique d'une légitimation », Argos, ${ }^{\circ} 5$ (1990), p. 52-64.

2. Les travaux de Marc Soriano sur la littérature enfantine (Guide de la littérature enfantine en 1959 puis surtout son Guide de la littérature pour la jeunesse en 1975) et plus particulièrement ses travaux sur Perrault (1968) et Jules Verne (1978) ont ouvert la voie. À partir de 1969, Isabelle Nières fait de la littérature pour la jeunesse un objet d'enseignement à l'université de Rennes II et publie de nombreux articles (citons «Didactisme et censure dans la littérature enfantine» (1972), «Recherches sur la littérature enfantine» (1972) ou «Les livres pour enfants et l'adaptation» (1974) avant de soutenir sa thèse en 1989 sur Lewis Carroll en France (1870-1985) : les ambivalences d'une réception littéraire. Citons plus récemment: Isabelle Nières-Chevrel, Littérature de jeunesse, incertaines frontières (2008) et Introduction à la littérature de jeunesse (2009); Jean Perrot, Jeux graphiques dans l'album pour la jeunesse (1991), Jeux et enjeux du livre d'enfance et de jeunesse (1999) et Carnets d'illustrateurs (2000); ou encore le Dictionnaire du livre de jeunesse : la littérature d'enfance et de jeunesse en France, I. Nières-Chevrel et J. Perrot dir. (2013).

3. Recto/Verso, $\mathrm{n}^{\circ}$ 4, « Mauvais genres », janvier 2009.

4. Genesis, ${ }^{\circ}$ 43, «Bande Dessinée», Pierre-Marc de Biasi et Luc Vigier (dir.), 2016.

5. Michael Nerlich, «Qu'est-ce qu'un iconotexte? Réflexions sur le rapport texte-image photographique dans La femme se découvre d'Évelyne Sinassamy», dans A. Montandon (dir.), Iconotextes : actes du colloque international tenu à l'Université Blaise Pascal, Clermont-Ferrand, 17-19 mars 1988, Paris, Ophrys, 1990, p. 255-302.

Genesis 48, 2019 
en opus uniques ou en séries. Protéiforme, la littérature pour la jeunesse échappe à toute forme de catégorisation reposant sur une généralisation et n'offre donc pas au généticien des avant-textes de nature différente de ceux des ouvres destinées à un lectorat adulte ou lues par celui-ci.

La littérature pour la jeunesse ne se définit que par son lectorat, trait définitoire ambigu et instable: d'une part, les lectorats effectifs changent, qu'un roman pour adultes soit adopté par les jeunes lecteurs et devienne un classique de la littérature pour enfants (par exemple David Copperfield ou encore Notre-Dame de Paris), ou qu'une æuvvre écrite à l'origine pour des enfants soit rééditée sous une couverture plus neutre, destinée aux adultes, comme ce fut le cas de la série des Harry Potter. D'autre part, l'adulte reste souvent présent comme médiateur entre l'œuvre et l'enfant : ce sont les adultes - éditeurs, bibliothécaires, parents, enseignants, conteurs, didacticiens... - qui décident des collections dans lesquelles les textes sont publiés, établissent des listes de lecture et conseillent les jeunes lecteurs, lisent ou racontent pour eux. Par ailleurs, ce qui faisait la spécificité de la littérature pour la jeunesse à ses débuts, c'était une intention didactique explicite à visée moralisatrice - que l'on songe au Télémaque de Fénelon (1694) ou encore au Little Pretty Pocket Book de Newbery (1744); cependant, si cette tendance n'a pas complètement disparu de la production littéraire, elle n'en est plus du tout la règle aujourd'hui.

La définition de ces auvres par un seul de leurs lectorats potentiels tend à réduire ces dernières à une "sous-littérature ». Il n'est ainsi pas rare que des auteurs s'opposent à la mention "pour la jeunesse» : Ramona Badescu, par exemple, auteur de la série Pomelo, refuse que soit indiqué sur ses livres l'âge préconisé du lecteur. Beaucoup défendent également l'idée que la catégorie "littérature jeunesse» n'a pas lieu d'être hors de considérations de marketing ou d'études sur l'histoire du livre. Pourtant, "la distinction entre enfant et adulte, leur opposition et leur irréductible distance 6 » résistent et s'imposent par une forme d'évidence. Nathalie Prince relève ainsi certaines spécificités : le jeu sur l'image et le support, le double lectorat, le "jeu d'architextes, d'archétypes, de mythes et de stéréotypes ${ }^{7}$ », sans qu'aucune de ces caractéristiques ne soit à elle seule définitoire de l'objet.

Une approche génétique de ces corpus n'échappe donc pas à la question à laquelle se heurtent tous les chercheurs qui se sont attachés à définir une poétique de la littérature pour la jeunesse : cette littérature existe-t-elle? En quoi consiste-t-elle? "Tous les ouvrages de l'esprit contiennent en eux-mêmes l'image du lecteur auquel ils sont destinés 8 »écrivait Sartre dans Qu'est-ce que la littérature? (chapitre «Pour qui écrit-on?»). La question du destinataire comme clef de lecture du processus créatif se pose avec une acuité particulière dans l'étude de genèses d'œuvres pour enfants parce que ce sont les seuls textes écrits, publiés et achetés par une population - les adultes - différente de celle de ses lecteurs premiers. Cette question n'est pour autant pas spécifique elle non plus à ces ouvres et l'on aurait tort de considérer que tout auteur envisage par défaut un lecteur semblable à lui-même. La littérature pour la jeunesse offre ainsi un terrain de choix pour que la génétique se penche sur la question des modes et des moments d'adresse au lectorat. Dans le même temps, si la

6. Nathalie Prince, La Littérature de jeunesse. Pour une théorie littéraire, Paris, Armand Colin, 2010, p. 129. 7. Ibid., p. 194.

8. Jean-Paul Sartre, Qu'est-ce que la littérature?, ch. «Pour qui écrit-on?», Paris, Gallimard, 1975, p. 119. 
nature des dossiers génétiques ou des processus créateurs en jeu dans la littérature pour la jeunesse n'est pas différente d'autres dossiers génétiques, la prise en compte du lectorat par le généticien ne devra pas pour autant devenir la seule clef d'interprétation des faits génétiques au détriment d'autres interprétations possibles, en particulier dans ce que Rudolf Mahrer appelle les "phases privées de la genèse ${ }^{9}$ », dans lesquelles précisément l'auteur ne communique pas les raisons de ses choix. C'est cette question de la place du lectorat dans la genèse de l'œuvre qu'abordent, dans ce numéro, Catherine Boré à propos des Malheurs de Sophie et Annie Tanguay à propos d'un recueil de la poétesse canadienne Louise Dupré.

Outre cet enjeu génétique, l'étude d'œuvres pour la jeunesse a des enjeux didactiques et de valorisation qui lui sont propres. L'expertise du généticien met en effet à la disposition d'un public non expert des manuscrits nouveaux, abordables par de jeunes lecteurs et permet aux didacticiens ou aux enseignants non spécialistes de s'appuyer sur des travaux génétiques de qualité qu'ils pourront à leur tour didactiser afin de rendre le processus créatif accessible à des apprentis écrivains. C'est aussi là un argument pour convaincre des auteurs d'accepter le regard inquisiteur et rationalisant du généticien sur leur propre travail. C'est ainsi, par exemple, qu'Agnès Rosenstiehl conçoit l'intérêt d'une analyse de ses avant-textes.

\section{La littérature pour la jeunesse : une écriture toujours collaborative?}

Nicolas Donin et Daniel Ferrer, à la suite de Jack Stillinger ${ }^{10}$, ont mis en évidence la banalité des cas d'auctorialité multiple dans un numéro de Genesis consacré aux genèses collaboratives ${ }^{11}$. Si des facteurs culturels complexes expliquent la pudeur de mise en France sur les contributions des proches de l'écrivain, éditeurs ou agents littéraires, "l'importance et la légitimité relatives des interventions éditoriales sont mieux admises à propos d'écrivains mineurs, ou travaillant dans des genres considérés comme mineurs, comme la littérature pour la jeunesse 12 ». C'est donc à leur statut d' « infralittérature » que l'on doit la conservation des documents attestant des interventions des différents acteurs de l'édition jeunesse. Plusieurs archives de maisons d'édition pour la jeunesse sont ainsi consultables à l'IMEC et les éditions Gallimard, Hachette ou Flammarion ont pu faire l'objet de publications ${ }^{13}$.

Si les interventions éditoriales ne sont pas l'apanage des éditeurs jeunesse, le généticien pourra cependant se demander dans quelle mesure les différents acteurs de la genèse considèrent eux aussi les textes pour la jeunesse comme mineurs et en quoi cela peut jouer dans leurs rapports de force, dans les modalités de leurs collaborations et dans les types d'interventions à l'œuvre dans les brouillons. En effet, les acteurs des genèses jeunesse

9. Rudolf Mahrer, «Anecdotique», Genesis, n 44, «Après le texte», 2017, p. 9.

10. Jack Stillinger, Multiple Authors and the Myth of the Solitary Genius, New York, Oxford University Press, 1994. 11. Genesis, n 41, «Créer à plusieurs mains », 2015. Voir aussi Recto/Verso, n 3, «La création en collaboration», juin 2008.

12. Nicolas Donin et Daniel Ferrer, «Auteur(s) et acteurs de la genèse », Genesis, n 41, op. cit., p. 14

13. Voir parmi d'autres : Alban Cerisier et Jacques Desse, De la jeunesse chez Gallimard : 90 ans de livres pour enfants, Paris, Gallimard/Chez les libraires associés, 2008; Jean-Yves Mollier, Louis Hachette (1800-1864) : le fondateur d'un empire, Paris, Fayard, 1999; ou Claire Delbard, Le Père Castor en poche (1980-1990) ou comment innover sans trahir?, Paris, L'Harmattan, 2007. À propos d'autres maisons d'édition : Matthieu Letourneux et Jean-Yves Mollier, La librairie Tallandier : histoire d'une grande maison d'édition populaire (1870-2000), Paris, Nouveau Monde Éditions, 2011. 
- éditeurs, traducteurs autant qu'auteurs - invoquent régulièrement sans ambages leur connaissance supposée des goûts et des souhaits du jeune lecteur, là où l'édition adulte pilote les choix de ses lecteurs de façon moins avouée par le péritexte et les paratextes auctoriaux, éditoriaux et critiques. En littérature pour la jeunesse, la nécessité de protéger le lecteur, de l'éduquer, voire de le contrôler est revendiquée par les éditeurs, qui en même temps doivent prendre en compte la nécessité de plaire aux acheteurs (parents, enseignants, prescripteurs), qui ne sont pas (toujours) les lecteurs. La génétique invite le critique à se méfier de ses propres présupposés, qui peuvent le conduire à minimiser certains phénomènes éditoriaux ou à l'inverse à construire toute intervention éditoriale comme une censure insupportable qu'il convient de réparer : William Butcher, par exemple, loin d'accepter les interventions d'Hetzel, propose l'édition, problématique, d'une version «dé-hetzélisée » des romans de Jules Verne 14.

Lors de la traduction, cette autre forme de réécriture, il est fréquent qu'éditeurs et traducteurs s'autorisent des modifications majeures sur ces textes dits mineurs, sous prétexte d'une meilleure connaissance du lectorat. Selon Godelène Logez, la traduction pour la jeunesse est "cibliste» car "plus que tout autre forme de traduction, elle est axée sur le destinataire 15 ». La célèbre série d'Enid Blyton, Le Club des Cinq, a subi, lors de sa réédition à partir de 2006 par la Bibliothèque Rose et Verte, de nombreux remaniements : sur les titres - Le Club des Cinq et les saltimbanques devenant Le Club des Cinq et le Cirque de l'Étoile pour éviter les controverses -; sur les temps du récit, où le présent supplante le passé simple; sur la syntaxe, où «on» vient remplacer «nous »; sur la structure même du récit avec la suppression de descriptions, jugées trop longues, ou de répliques de personnages, ainsi que de certains éléments de l'intrigue, comme la rencontre du forain nommé Pancho dans l'épisode cité. L'hérö̈ne d'Astrid Lindgren, Fifi Brindacier, s'était, elle, considérablement assagie dans les premières traductions françaises ${ }^{16}$. Plus récemment, la première traduction française de l'album de Julia Donaldson et Axel Scheffler, The Gruffalo, non seulement ne respectait pas la versification et le rythme du texte, mais dénaturait le rapport texte-image en modifiant considérablement la mise en page ${ }^{17}$, ce qui est d'ailleurs souvent le cas lors des rééditions d'œuvres pour la jeunesse 18 .

Par ailleurs, l'équipe éditoriale peut être un partenaire indispensable, qui encourage, suggère, relance l'inspiration, comme le montre, dans l'étude ci-dessous sur la genèse de Fantastic Mr Fox, la contribution des agents littéraires et éditeurs successifs de Roald Dahl. Il est d'ailleurs révélateur qu'une même personne puisse être auteur, illustrateur et

14. William Butcher, Jules Verne inédit : les manuscrits déchiffrés, Lyon, ENS éditions/Institut d'histoire du livre, 2015, p. 455. Voir le compte rendu de Philippe Scheinardt dans Genesis, n 42, 2016.

15. Godelène Logez, «L'union des contraires... Quelques réflexions à partir de la traduction de deux romans de Joan Lingard», dans F. Antoine (dir.), «Traduire pour un jeune public », Ateliers 27, Villeneuve-d'Ascq, Université Charles de Gaulle-Lille 3, 2001, p. 54

16. Christina Heldner, «Une anarchiste en camisole de force; Fifi Brindacier ou la métamorphose française de Pippi Langstrump», La Revue des livres pour enfants, $\mathrm{n}^{\circ}$ 145, printemps 1992, p. 65-71.

17. Julia Donaldson et Axel Scheffler, Le Gruffalo, Paris, Gallimard Jeunesse et Éditions Autrement, 1999. L'album ayant eu le succès que l'on sait, une nouvelle traduction, par Jean-François Ménard, a été publiée chez Gallimard Jeunesse en 2013 dans une mise en page respectant l'original. Remerciements à Françoise Thau-Baret qui nous a fait découvrir ces deux traductions.

18. Sur l'analyse de tels phénomènes de réécriture par la traduction, voir Virginie Douglas et Florence Cabaret, La Retraduction en littérature de jeunesse, Bruxelles, P.I.E. Peter Lang, 2014. 


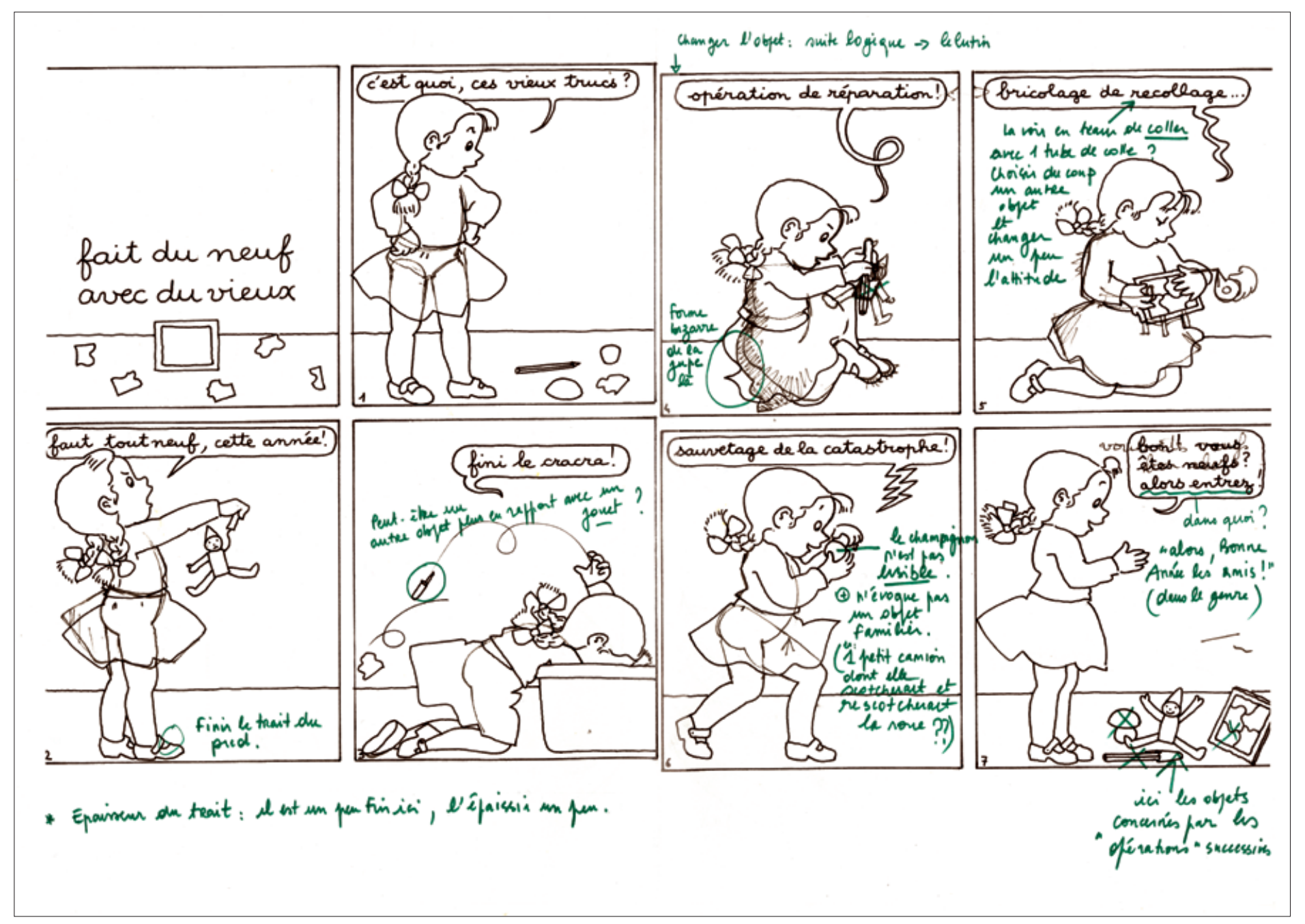

Fig. 1 : Agnès Rosenstiehl, Mimi Cracra fait du neuf avec du vieux. Format : $42 \mathrm{~cm} \times 29,7 \mathrm{~cm}$. Dossier "Mimi Cracra", 378RSN/24/0, IMEC.

(๑) Archives Agnès Rosenstiehl.

responsable de collections, ces différentes activités contribuant toutes selon des modalités différentes à la création, comme l'a montré Cécile Vergez-Sans dans son étude des carnets d'Olivier Douzou ${ }^{19}$. Cette complicité entre auteur-illustrateur et éditeur se retrouve dans la genèse des 250 épisodes de Mimi Cracra écrits par Agnès Rosenstiehl pour le journal Pomme d'Api à destination des 3-7 ans (1976-2005). L'éditrice apparaît comme la première lectrice de l'œuvre. Dans l'épisode Mimi Cracra fait du neuf avec du vieux (fig. 1), par exemple, l'éditrice annote scrupuleusement tout ce qui la dérange dans le manuscrit et semble gêner la réception de l'histoire et la lecture de l'image. Par exemple, sur l'illustration: "forme bizarre de la jupe là» (page 4) ou «finir le trait du pied» (page 2); sur la prise en compte du public-cible et de son univers de référence : elle demande à l'auteure de remplacer un «champignon», jugé «non lisible» pour le lecteur, par un «objet familier, ex. : 1 petit camion dont elle [Mimi Cracra] scotcherait et rescotcherait la roue?? » (page 6) ou encore

19. Cécile Vergez-Sans, «Les carnets d'Olivier Douzou, création et travail d'édition», dans Littérature de jeunesse : la fabrique de la fiction, P. Clermont et D. Henky (dir.), Francfort-sur-le-Main, Peter Lang, 2017, p. 143-159. 
un stylo-plume sorti d'un coffre par « un autre objet plus en rapport avec un jouet » (page 3), demande formulée sous la forme interrogative ( peut-être...?»).

Le rôle de la commande est particulièrement frappant dans l'écriture des séries et des cycles, qui, de Babar au Club des Cinq, de Petit Ours Brun à Sans Atout et des Petites Filles de la Comtesse à Harry Potter, constituent une part importante de l'édition pour la jeunesse20. Une génétique de l'écriture du cycle, telle que l'ont dessinée Alain Pagès et Olivier Lumbroso dans le numéro 42 de Genesis 21 , pourrait ainsi viser une étude comparative des auvres pour la jeunesse et des séries et cycles dont le lectorat n'est pas explicitement indiqué.

\section{Iconotextes et jeux : des collaborations créatrices à la collaboration avec le lecteur}

Si les corpus jeunesse sont un terrain de choix pour l'étude des collaborations dans la genèse éditoriale, c'est aussi parce que les ouvres pour la jeunesse sont presque toujours, de l'album sans texte au roman à peine illustré, des iconotextes ${ }^{22}$, présentant des contraintes de forme spécifiques. L'album a ainsi souvent été considéré comme la forme par excellence des livres pour jeunes enfants 23 . L'attention du jeune lecteur semble en effet nécessiter, pour être soutenue, la présence d'éléments visuels, qu'il s'agisse d'images ${ }^{24}$ ou, a minima, lorsque l'histoire est racontée, d'une incarnation par le corps et la voix du conteur. On pourrait donc identifier à bien des égards la génétique de la littérature pour la jeunesse à celle de la bande dessinée, explorée dans le numéro 43 de Genesis, et adopter la modélisation proposée par Pierre-Marc de Biasi 25.

Tous les cas de figure se rencontrent dans l'élaboration collaborative du texte et de l'image. Certains sont auteurs et font illustrer leurs ouvres par d'autres (Thomas Scotto), d'autres sont auteurs et illustrateurs de leurs propres œuvres, comme Claude Ponti (qui a illustré pour d'autres de façon extrêmement marginale), d'autres illustrent à la fois leurs propres textes et ceux d'autrui (Olivier Douzou, Christian Voltz), ou combinent les trois configurations, comme Rascal, qui a écrit par exemple Ami-Ami illustré par Stéphane Girel (Pastel, 2002), illustré

20. Voir, entre autres, Anne Besson, D'Asimov à Tolkien, cycles et séries dans la littérature de genre, Paris, CNRS Éditions, 2004 et «Du Club des Cinq à Harry Potter, cycles et séries en littérature de jeunesse contemporaine», dans La Littérature de jeunesse en question(s), N. Prince (dir.), Presses universitaires de Rennes, 2009, p. 117-154. 21. Olivier Lumbroso et Alain Pagès, «Pour une génétique des cycles romanesques », Genesis, n 42, 2016.

22. L'inverse n'est pas vrai, puisque toutes les formes d'iconotextes : albums, romans graphiques, bandes dessinées, etc. existent dans la littérature «pour adultes ».

23. Voir l'étude qu'y a consacré Sophie Van der Linden : Lire l'album, Le Puy-en-Velay, L'atelier du poisson soluble, 2006.

24. L'album de B.J. Novak, The Book with No Pictures (2014) confirme la règle, puisque ce sont les mots qui deviennent des images, par le jeu sur les polices de caractères et les couleurs.

25. Pierre-Marc de Biasi, «Pour une génétique de la bande dessinée», Genesis, n 43, «Bande dessinée», P.-M. de Biasi et L. Vigier (dir.), 2016; et dans le même numéro, Pierre-Marc de Biasi et Luc Vigier, «Un nouvel horizon génétique : la bande dessinée ?». Citons ici aussi les travaux pionniers de Jean Perrot sur les genèses d'illustrations dans Carnet d'illustrateurs, Paris, Éditions du Cercle de la librairie, 2000; et la thèse de Christine Plu, Georges Lemoine : illustrer la littérature au XXe siècle, soutenue en 2005 à l'université de Rennes 2 sous la direction d'Isabelle Nières-Chevrel. 
Nos amies les bêtes pour Léo Rau (Pastel, 2011) et publié des auvres dont il est auteur et illustrateur, Le vent m'a pris (Pastel, 2004). Les temporalités varient : tel texte est illustré après son achèvement, comme ce fut le cas des romans de Mohammed Dib et de Jean-Pierre Robert évoqués dans le présent numéro, illustrés par plusieurs artistes différents choisis par les éditeurs à l'occasion de rééditions successives sans qu'auteurs et illustrateurs ne se soient jamais rencontrés. Tel autre est illustré en même temps qu'il s'écrit, comme ce fut le cas du roman Sylvie and Bruno de Lewis Carroll, illustré simultanément par Harry Furniss 26. Carroll est allé jusqu'à graver lui-même une série de blocs pour illustrer Alice, et comme lui, Roald Dahl essaye d'abord d'inventer simultanément texte et images pour Fantastic Mr Fox, avant de confier les illustrations à Donald Chaffin. Dahl sera toujours activement impliqué dans le choix des artistes qui illustreront ses æuvres, mais sans véritablement intervenir dans leur travail jusqu'à la rencontre avec Quentin Blake, avec qui il construira progressivement une véritable collaboration. Les genèses d'auteurs-illustrateurs présentées ici, qu'il s'agisse d'Agnès Rosenstiehl ou d'Yvan Pommaux, montrent le développement simultané du texte et de l'image plutôt que des étapes successives.

Le choix du support étant primordial, comme le souligne Nathalie Prince 27 , les collaborations s'étendent aux coloristes, graveurs et imprimeurs. Au-delà des livres-jouets ou livres-objets de tissu ou de plastique imperméable et du cas particulier des livres pop-up, le livre pour enfants doit être adapté aux mains de l'enfant et prompt à attiser sa curiosité. Dans son étude sur Le Père Castor en poche (1980-1990)28, Claire Delbard a montré ainsi le rôle pionnier du lancement des Albums du Père Castor par Paul Faucher. Les auteurs sont amenés à expérimenter des formats différents, comme Claude Ponti 29 , ou à jouer sur la disposition des illustrations, comme lorsque le lecteur de Lewis Carroll en tournant la page fait traverser le miroir à Alice, transforme le bébé en porcelet ou fait disparaître le chat du Cheshire, excepté son sourire ${ }^{30}$. En 1877 déjà31, Lewis Carroll se préoccupait ainsi de la solidité des reliures, souhaitant que de très jeunes enfants puissent malmener à loisir ses livres, et en avait choisi la couleur, rouge, pour son attractivité supposée aux yeux des enfants. Comme Hervé Tullet aujourd'hui, qui invite les lecteurs de Un livre ou de Couleurs 32 à secouer ou à pencher le livre pour observer à la page suivante les effets produits par ce mouvement, le narrateur de The Nursery Alice suggère au lecteur, dans le premier chapitre, de secouer le livre pour faire trembler de peur le lapin blanc.

26. Voir Christine Collière-Whiteside, «Illustrating the Sylvie and Bruno books: the collaboration of Lewis Carroll and Harry Furniss », Recto/Verso, n 3, juin 2008.

27. Nathalie Prince, La Littérature de jeunesse. Pour une théorie littéraire, Paris, Armand Colin, 2010, p. 159 sq. 28. Claire Delbard, Le Père Castor en poche (1980-1990) ou comment innover sans trahir?, Paris, L'Harmattan, 2007.

29. Adèle de Boucherville, La Fabrique de Claude Ponti, Le Puy-en-Velay, L'atelier du poisson soluble, 2016.

30. On notera que de nombreuses rééditions d'Alice's Adventures in Wonderland et de Through the Looking-Glass ont négligé de placer les paires d'illustrations de Tenniel dos à dos au recto et au verso d'une même page, perdant ainsi cet effet.

31. «The essential point is that the books shall be able to stand an exceptional amount of knocking about without coming to pieces », lettre à Macmillan du 15 décembre 1877, reproduite dans Lewis Carroll and the House of Macmillan, Morton N. Cohen et Anita Gandolfo (dir.), Cambridge, Cambridge UP, 1987, p. 139.

32. Hervé Tullet, Un livre, Paris, Bayard jeunesse, 2010, et Couleurs, Paris, Bayard, 2014. 
Le jeu, en effet, entre directement dans la genèse des textes pour la jeunesse : c'est ainsi qu'Yvan Pommaux construit en Lego le décor de la série de BD Les Enquêtes de Théo Toutou avant de le dessiner ${ }^{33}$ et que les objets bricolés de Christian Voltz ${ }^{34}$ ou de Claude Ponti 35 jouets, figurines, scènes qui deviendront des illustrations - s'apparentent au jeu symbolique et au faire-semblant. En léguant à la ville de Strasbourg non seulement ses archives, mais aussi sa collection de jouets, Tomi Ungerer invite le généticien à considérer les jeux de simulacre, de «mimicry» selon le terme de Roger Caillois, comme partie prenante de la genèse des histoires ${ }^{36}$ : la création se déploie sur le mode du conditionnel enfantin, le "on dirait qu'on serait...» ou de l' «imparfait préludique» («On était sur une île et toi, tu étais un naufragé...»). Si les cas de livres pour la jeunesse où l'âge de l'auteur coüncide avec celui des lecteurs restent rares, comme Eragon, publié à 19 ans par Christopher Paolini, de nombreux classiques sont connus pour avoir trouvé leur origine dans des lettres adressées à des enfants, comme les contes de Beatrix Potter ou les aventures de Bilbo le Hobbit, dans une improvisation orale partagée, comme Alice's Adventures in Wonderland, les Just So Stories de Kipling ou The Wind in the Willows, ou dans des jeux partagés, à l'instar des jeux entre J. M. Barrie et les enfants Llewelyn Davies qui ont inspiré les aventures de Peter Pan ou des histoires attribuées aux peluches de Christopher Robin Milne qui deviendront les personnages de Winnie the Pooh. La participation d'enfants à la genèse d'une cuvre devient un gage d'authenticité désirable, au point qu'Henri Bosco, par exemple, a inventé de toutes pièces la légende selon laquelle ses élèves lui auraient dicté au moins un tiers de L'Âne Culotte 37. L'institution favorise cependant aussi les rencontres entre auteurs et élèves dans le cadre d'ateliers d'écriture donnant lieu à de véritables pratiques collaboratives, comme en atteste la genèse du livre de Thomas Scotto, Le Duel des frères Flint, qui trouva ses origines dans un projet de rencontre et de correspondance avec une classe de fin de primaire 38 . Le rapport à l'enfance qui se construit ainsi dans l'écriture demande à être interrogé, lorsque des traces existent de ces phases de la genèse.

\section{La prise en compte du (double) lectorat}

Si écrire pour la jeunesse, c'est écrire pour la part d'enfance qu'il y a en nous, le texte pour la jeunesse s'adresse régulièrement à un double lectorat, adulte et enfant, qui se concrétise dans le dispositif particulier de la lecture à voix haute à l'enfant non lecteur. Claude Ponti prend ainsi un malin plaisir à glisser dans les images mille détails à l'intention des enfants, que l'adulte, occupé par le texte, ne remarquera pas, ou pas immédiatement,

\footnotetext{
33. Conversation privée entre Solène Audebert-Poulet et Yvan Pommaux. La construction en Lego, ainsi que les croquis et le dessin sont reproduits dans Yvan Pommaux : correspondance entre Yvan Pommaux et Lucie Cauwe, Paris, L'école des loisirs, coll. «Tout sur votre auteur préféré», 2014.

34. Cf. dans ce numéro la rubrique «Inédits », Karine Meshoub-Manière, «Christian Votlz, Heu-reux».

35. Adèle de Boucherville, op. cit.

36. Évelyne Bedoin, «La collection de jouets Tomi Ungerer, une archive de l'œuvre?», dans P. Clermont et D. Henky (dir.), Littérature de jeunesse : la fabrique de la fiction, Francfort-sur-le-Main, Peter Lang, 2017, p. 61-78. 37. Sandra L. Beckett, De grands romanciers écrivent pour les enfants, Montréal, Presses universitaires de Montréal, 1997, p. 278.

38. Juliette Burat, «De la rencontre avec un auteur de littérature jeunesse à la production d'écrit littéraire en cycle 3 », dans M. Jacques et C. Raulet-Marcel (dir.), Inventions de l'écriture, Dijon, EUD, 2014, p. 213-229.
} 
tandis que d'autres auteurs multiplient à l'intention des adultes des clins d'œil que le jeune lecteur aura le plaisir de décoder lorsqu'il sera plus grand. Le décalage entre l'histoire racontée par le texte et lue par l'adulte et les indices présents dans les images, qui racontent une autre histoire, est caractéristique de l'album, même s'il n'est pas systématique.

Il reste cependant délicat, sauf déclaration explicite de la part de l'auteur, d'attribuer à la seule anticipation de ce double lectorat les phénomènes de décalage entre texte et image, qui peuvent relever d'une logique intrinsèque à l'œuvre. De même, dans les phases privées et pré-éditoriales de la genèse, lorsque le jeu des collaborations ne donne plus à voir les questionnements de l'auteur, comment affirmer que telle rature atteste d'une volonté de lisibilité, de simplification, que tel choix d'écriture répond au besoin de satisfaire les horizons d'attente supposés d'un lecteur particulier? Les notes expliquant au lecteur des éléments du contexte historique de La Chanson de Hannah sont-elles le fait de l'auteur, Jean-Paul Nozière, ou de son éditeur, et à quel moment de la genèse sont-elles apparues? De telles explicitations, si elles sont intégrées au corps du texte, doivent-elles être attribuées à l'anticipation de l'ignorance du jeune lecteur ou à un choix d'écriture? Dans quelle mesure le travail du style mis en évidence par Cyrille François ${ }^{39}$ dans les premiers contes d'Andersen est-il révélateur d'une prise en compte du lecteur projeté ou de l'émergence d'un style propre? Se pencher sur les brouillons d'écrivains pour la jeunesse permet de (re)mettre le lecteur visé plus ou moins consciemment par l'auteur au centre du processus créatif, d'en étudier le rôle moteur(?) dès les phases pré-éditoriales, notamment quand il s'agit d'un lecteur particulier, aux compétences de lecture encore en construction, voire à peine naissantes.

Le cas des ouvres réécrites pour un public différent ouvre des possibilités d'analyses comparatives. Dirk Van Hulle, Vincent Neyt et Vanessa Joosen étudient dans le présent numéro les réécritures successives des contes des frères Grimm, montrant comment des textes issus de la tradition orale, publiés à l'intention d'un lectorat adulte, ont été réécrits au fur et à mesure qu'ils entraient dans le canon des textes pour enfants. Tournier, lui, a toujours affirmé que Vendredi ou les Limbes du Pacifique n'était qu'un "brouillon 40 » de Vendredi ou la vie sauvage, qui est un meilleur état du texte, "plus achevé, plus serré, beaucoup moins philosophique, beaucoup moins abstrait et tout aussi riche. [...] C'est une seconde version et qui est si bonne que les enfants peuvent la lire 41 ». Ce refus de l'étiquette «jeunesse » peut être une façon d'éviter les «murs de censure » qui peuvent se dresser :

Parce qu'on vous dit : «Mais voyons, un livre pour enfants, il faut éviter le sexe, il faut éviter l'argent, il faut éviter la politique. [...].» Tout le monde sait que les enfants n'ont ni sexe, ni politique, ni argent ${ }^{42}$.

C'est aussi tout simplement admettre qu'aucun livre n'a un lectorat unique et homogène : à Genette qui évoque une double réception de Vendredi, "Je suis seul devant ce texte, et pourtant je me sens deux : l'enfant qu'il vise et l'adulte qu'il atteint», Tournier répond

39. Cyrille François, «Andersen trouve-t-il son conte? De «Dødningen» ("Le mort", 1830) à "Reisekammeraten" ("Le compagnon de voyage", 1835)», Genesis, n 44 «Après le texte. De la réécriture après publication», 2017, p. 65-79.

40. Cité dans Sandra L. Beckett, De grands romanciers écrivent pour les enfants, op. cit., p. 193.

41. «Questions à Michel Tournier», dans Sandra L. Beckett, op. cit., p. 266.

42. «Entretien du 25 mai 1994 avec Michel Tournier», dans Sandra L. Beckett, op. cit., p. 275. 
ainsi que Genette "n'est pas un lecteur moyen et représentatif43». Écrire pour la jeunesse s'apparente-t-il donc simplement à viser un idéal littéraire de "limpidité, de brièveté et de proximité du concret 44 », ou à une contrainte oulipienne, comme la limite des 250 mots les plus courants respectée par Dr Seuss dans l'écriture de The Cat in the Hat, ou les consignes reçues par Marie-Aude Murail, Élisabeth Brami ou René Escudié lorsqu'ils écrivent des textes pour des manuels d'apprentissage de la lecture 45 ? Le statut de la littérature pour la jeunesse est tel qu'il est impossible de s'appuyer sur les déclarations des auteurs pour dégager une éventuelle spécificité de la prise en compte du lectorat dans la genèse, et tout un champ d'études génétiques s'ouvre là : les brouillons de Yourcenar nous diront peut-être comment elle a réécrit Comment Wang-Fô fut sauvé pour un public plus large, comment Tove Jansson a réécrit pour des lecteurs plus âgés au contraire quatre des histoires des Moomins, ou comment Dahl a transformé la nouvelle "The champion of the world» en un roman pour enfants, Danny the Champion of the World. Les corpus génétiques d'auteurs qui écrivent pour différents publics, comme Élisabeth Brami ou Jean-Paul Nozière, sont à ce titre particulièrement prometteurs.

\section{Les archives des auteurs pour la jeunesse : conservation, valorisation, exploitation didactique}

Les archives génétiques des ouvres de littérature pour la jeunesse se caractérisent, elles aussi, par l'existence d'un public spécifique qui s'ajoute aux amateurs éclairés et aux généticiens : d'une part, l'attractivité des images, le côté spectaculaire de certains brouillons illustrés et la nostalgie des lectures d'enfance permettent de développer des projets d'une nature et d'une ampleur inhabituelles en génétique. Citons le succès des expositions itinérantes de The House of Illustration 46 ou le Musée de l'illustration jeunesse à Moulins ${ }^{47}$. D'autre part, les enfants et les enseignants représentent un public qu'on pourrait dire «captif», pour lequel de nouvelles modalités de valorisation se développent, car ce public non spécialiste, qu'il s'agisse des élèves ou des professeurs des écoles, a des besoins spécifiques : les brouillons revêtent pour lui un enjeu didactique fort, mais il est nécessaire de lui proposer un accompagnement pour qu'il sache comment aborder le brouillon et le discours génétique qui l'entoure.

Si Luc Vigier a pu parler à propos de la BD de "génétique à ciel ouvert 48 », il en va souvent de même pour la littérature jeunesse. Que ce soit dans la collection «la fabrique 49 »

43. Ibid., p. 276.

44. Ibid., p. 273.

45. Ainsi, le manuel Bulle (Bordas), construit autour d'un roman épistolaire d'Élisabeth Brami, ou Abracadalire (Hatier), qui s'appuie sur cinq albums de Fanny Joly et Pierre Élie Ferrier (Pef), René Escudié et Lionel Labeyrie, Nicolas Hirsching et Denise Chabot, Stephan Lévy-Kuentz et Jean-Marie Renard, Élisabeth Brami et Maurice Rosy. 46. <https://www.houseofillustration.org.uk/>.

47. Musée de l'illustration jeunesse (MIJ) : <http://musees.allier.fr/>.

48. Luc Vigier, «Bande dessinée : une génétique à ciel ouvert», Genesis, n 43, 2016, p. 51-63.

49. Adèle de Boucherville, La Fabrique d'Olivier Douzou, Le Puy-en-Velay, L'atelier du poisson soluble, 2015,

et La Fabrique de Claude Ponti, op. cit., 2016. Un ouvrage sur Bruno Heitz est en préparation. 
(L'atelier du poisson soluble), à travers des expositions ou des livres ${ }^{50}$, sur des blogs ou des sites personnels, des auteurs ont ainsi tenté de rendre accessibles «leur caisse à outils» (Philippe Barbeau ${ }^{51}$ ) de façon plus ou moins extensive. Ce geste peut se lire comme une façon, pour ces auteurs, de revendiquer leur appartenance à la Littérature, en montrant le travail réalisé. Ce même geste témoigne aussi de leur volonté de partager, en particulier avec un jeune public, l'œuvre dans sa totalité, soit dans une perspective didactique, en lien avec des visites dans les écoles, soit pour établir un lien avec toute une communauté de lecteurs, notamment lorsque les auvres constituent une série dont les lecteurs attendent la suite. Un exemple particulièrement réussi est celui de la première version du site officiel de J. K. Rowling 52 , immersif et interactif, où était mis en scène sous forme de chasse au trésor le partage d'une douzaine d'avant-textes avec les lecteurs assez persévérants pour explorer le site dans ses moindres détails. L'auteur y rendait compte régulièrement de son travail d'écriture en cours, invitant ainsi les lecteurs dans l'espace intime de l'activité d'écriture, représenté en page d'accueil par le bureau en désordre d'un écrivain.

Cette pratique est surtout l'apanage des auteurs-illustrateurs, car les documents publiés sont le plus souvent des images - croquis, esquisses préparatoires, chemins de fer - plutôt que des brouillons textuels, sans doute parce qu'ils sont plus faciles d'accès, et donc plus propres à susciter l'intérêt du (jeune) public. Ainsi, François Place, auteur-illustrateur, ne propose que des dessins sous l'onglet «recherches» (ou «carnets ${ }^{53}$ ») de son site, alors que ses auvres comportent une part importante de texte. De même, le dossier de presse de l'exposition «Christian Voltz, poésie bidouillée 54 » ne mentionne aucun des brouillons qui faisaient pourtant partie des objets exposés. Significativement, la rubrique du site de Mario Ramos 55 permettant d'accéder à ses documents préparatoires s'intitule "croquis », bien qu'on y trouve un certain nombre de documents iconotextuels. Un récent entretien avec Axel Scheffler et Julia Donaldson ${ }^{56}$ publié dans The Guardian est accompagné d'une galerie de documents de travail de Scheffler commentés par l'artiste, mais là encore, aucun brouillon de l'auteure n'est proposé. L'habitude de mettre en valeur un portfolio d'œuvres par les artistes, habitude que n'ont pas les auteurs, peut expliquer ce phénomène : le blog «Picturebook Makers 57 » est composé de témoignages d'illustrateurs, mais aussi d'auteurs et auteurs-illustrateurs de toutes nationalités, comme par exemple Béatrice Alemagna (Italie), Kithy Crowther (Belgique) ou encore Jon Klassen (Canada), Chris Haughton (Angleterre),

50. Quentin Blake, Pages, mots, images, Paris, Gallimard, 2014, version retravaillée de La Vie de la page, Paris, Gallimard, 1995.

51. Voir le site < Philippe Barbeau, ma caisse à outils >, consulté le 10 février 2019.

52. Cette première version du site $<w w w . j k r o w l i n g . c o m>$ a fonctionné de 2004 à 2012 . Il a été recréé en 2018 dans son état d'origine et peut être consulté sur $<$ The Rowling Library $>$. Plusieurs des brouillons, dessins ou scénarios ainsi partagés ont été exposés à la British Library lors de l'exposition «Harry Potter: a History of Magic», octobre 2017-février 2018.

53. <https://www.francois-place.fr/carnets/>, consulté le 10 février 2019

54. Exposition à Lambersart de décembre 2016 à février 2017, voir < Christian Voltz, poésie bidouillée >, consulté le 10 février 2019.

55. <http://www.marioramos.be/>, consulté le 10 février 2019.

56. Lisa Allardice, «Julia Donaldson \& Axel Scheffler: "The Gruffalo's not a curse... it can be a burden"», The Guardian, 22 décembre 2017, consulté sur site le 10 février 2019.

57. < http://blog.picturebookmakers.com/>, consulté le 10 février 2019. 
Olivier Tallec (illustrateur français), richement illustrés de croquis, esquisses, chemins de fer, etc. qui documentent la genèse d'un de leurs albums. Julie Danielson, critique littéraire spécialisée dans la littérature pour la jeunesse, notamment pour la Kirkus Reviews, publie très régulièrement sur son blog personnel 58 des entretiens avec des illustrateurs ou auteursillustrateurs qui partagent à cette occasion divers documents génétiques.

Les documents de travail ainsi partagés ne sont que des échantillons, et non des dossiers génétiques complets, et ces livraisons font l'objet d'une sélection et d'une mise en scène par les auteurs ou leurs éditeurs. Des maisons d'édition comme L'école des loisirs s'associent à l'Éducation nationale pour proposer des animations pédagogiques où les écrivains témoignent de leur travail d'écriture 59 , animations qui donnent lieu à des entretiens et des documentaires vidéo ${ }^{60}$. La Charte des auteurs et illustrateurs jeunesse 61 facilite les interventions d'écrivains dans des classes sous diverses formes : rencontres, ateliers d'écriture, correspondances. Conscients de l'intérêt pédagogique ou génétique de ces avant-textes, les auteurs modifient parfois leurs pratiques : Jean-Paul Nozière avait ainsi choisi de changer de couleur lors d'une campagne de réécriture d'Un été algérien dans l'intention de faire apparaître plus clairement le travail de réécriture lorsqu'il montrerait son manuscrit à des élèves ${ }^{62}$. De même, Yvan Pommaux explique qu'après avoir livré à Solène Audebert-Poulet ses carnets et ses brouillons pour la thèse de doctorat qu'elle mène sur son travail d'écriture et la genèse de ses æuvres, il a tenté d'écrire son nouvel album « sans faire de brouillons 63 ». Il convient donc de lire ces témoignages avec précaution. Le partage de ces documents constitue toutefois une manne non négligeable pour les enseignants : c'est ainsi que nous avons pu trouver sur le site de Mario Ramos suffisamment de brouillons permettant de construire une séquence d'initiation à l'analyse génétique à l'intention d'élèves de CM1-CM2 et de professeurs des écoles stagiaires 64.

L'enseignant désireux de montrer, voire de faire étudier des brouillons d'écrivains à ses élèves de primaire, se heurte néanmoins à la difficulté de trouver des corpus exploitables d'un point de vue didactique : les dossiers jeunesse conservés dans des archives, plus complets, ont rarement fait l'objet d'études génétiques. Souvent, ces études ne sont pas accompagnées

58. «Seven Impossible Things before Breakfast», <http://blaine.org/sevenimpossiblethings/> consulté le 10 février 2019.

59. Par exemple, la rencontre organisée avec Yvan Pommaux par L'école des loisirs, le 14 mars 2018 à Douai.

60. Voir ainsi Yvan Pommaux au travail: La Commune, dans la collection des Grandes images de l'histoire, documentaire portant sur la genèse de l'album La Commune, des premiers avant-textes à l'impression finale, $<$ https:// www.ecoledesloisirs.fr/auteur/yvan-pommaux >.

61. <http://www.la-charte.fr/>.

62. Entretien lors de la journée d'études «Regards croisés sur les brouillons d'écrivains pour la jeunesse : de la génétique à la didactique», organisée par C. Collière-Whiteside, K. Meshoub-Manière, Université de Bourgogne Franche-Comté, le 27 mai 2016.

63. Animation pédagogique de Douai, cf. note 59.

64. Nous rendons compte de ce travail dans l'article suivant : Karine Meshoub-Manière et Christine CollièreWhiteside, «Réécrire des textes au cycle 3, repenser les processus d'écriture en master MEEF : une expérimentation croisée à partir de brouillons d'écrivains jeunesse », Repères, n 57, 2018, p. 99-122, <https://journals.openedition. org/reperes/1496>, consulté le 10 février 2019. 
de reproduction des manuscrits ${ }^{65}$. Les travaux pionniers de Claudette Oriol-Boyer 66 , qui a publié en 2002 une série de séquences pédagogiques proposant de faire observer aux élèves des manuscrits d'écrivains afin de travailler sur la réécriture, s'appuient sur des avant-textes de Flaubert, Zola, Maupassant, Ponge et de Jean Lahougue et sont destinés à des élèves du secondaire, tout comme les dossiers pédagogiques sur Queneau, Zola et Hugo associés par la Bibliothèque nationale de France à l'exposition virtuelle «Brouillons d'écrivains 67 » ou le site ArchiZ68. C'est tout l'enjeu de ce numéro de Genesis que de commencer à répondre aux besoins des didacticiens à la recherche de brouillons abordables par de jeunes élèves pour développer chez eux une posture d'auteur69. La partie Chroniques recense ainsi un certain nombre de fonds, dont certains s'attachent non seulement à conserver les archives à l'intention des chercheurs, mais aussi à trouver les moyens de les valoriser auprès d'autres publics. Rachel White, conservatrice aux archives du musée Roald Dahl, explique ainsi dans un entretien publié ici les stratégies imaginées pour mettre en valeur des brouillons textuels de Dahl ainsi que les projets pédagogiques développés en direction des écoles et des familles autour de l'écriture.

En imposant des contraintes particulières, le public enfantin oblige à trouver des stratégies innovantes de valorisation des manuscrits. La littérature pour la jeunesse constitue en cela un terrain d'expérimentation privilégié, dont les résultats pourraient en retour permettre de diffuser autrement des corpus plus confidentiels, qui pourraient eux aussi être ouverts à un public plus large, au-delà des généticiens avertis et des amateurs de littérature. Même si les objectifs didactiques diffèrent des objectifs génétiques, les archives d'avant-textes d'œuvres lues par de jeunes lecteurs pourraient s'inscrire tout naturellement dans un dialogue avec l'institution scolaire.

65. Marie-José Strich, «Critique génétique d'un manuscrit : La Bible d'une grand-mère (1869) », Cahiers Robinson, $\mathrm{n}^{\circ}$ 9, La Comtesse de Ségur et ses alentours, 2001, p. 183-192.

66. Claudette Oriol-Boyer (dir.), Critique génétique et didactique de la réécriture : travailler avec les brouillons d'écrivains, Paris, Bertrand-Lacoste, 2003.

67. Marie-Odile Germain et Danièle Thibault (dir.), «Brouillons d'écrivains », exposition à Bibliothèque nationale de France, 27 février-24 juin 2001. < http://editions.bnf.fr/brouillons-d\%C3\%A9crivains-0>, consulté le 10 février 2019.

68. Olivier Lumbroso, Jean-Sébastien Macke et Alain Pagès, «Le site ArchiZ», Genesis, n 42, 2016, p. 139-144. 69. Nous utilisons ici «posture d'auteur» dans le sens où l'entendent Catherine Tauveron et Pierre Sève, Vers une écriture littéraire ou comment construire une posture d'auteur à l'école de la GS au CM, Paris, Hatier, 2005. Il s'agit de faire de l'enfant non pas seulement un «scripteur» ou un «producteur» de textes respectant des normes, mais bien un «auteur», qui s'efforce d'inscrire un lecteur modèle comme partenaire au cœur de sa stratégie textuelle. 